\title{
Impacto na Aprendizagem Envolvendo o Desenvolvimento de Aplicativos na Educação Básica: um Mapeamento Sistemático da Literatura
}

\author{
Giselle Araújo e Silva de Medeiros - UFSC - giselle.medeiros@ posgrad.ufsc.br \\ Christiane Gresse von Wangenheim - UFSC - c.wangenheim@ufsc.br \\ Juliana Cristina Faggion Bergmann - UFSC - juliana.bergmann @ufsc.br
}

\section{Resumo}

O ensino de computação na Educação Básica tem se apresentado como uma alternativa para o desenvolvimento de habilidades do século XXI. Vem sendo tipicamente ensinado por meio de linguagens de programação como App Inventor, ferramenta que possibilita o desenvolvimento de aplicativos móveis para smartphones. Porém, ainda não se tem uma compreensão dos impactos que práticas pedagógicas têm gerado na aprendizagem dos alunos com esse enfoque específico no ambiente de programação. $\mathrm{O}$ artigo apresenta o resultado de um Mapeamento Sistemático da Literatura em que se buscou analisar os impactos de práticas pedagógicas de desenvolvimento de aplicativos com o App Inventor na aprendizagem de conteúdos curriculares da Educação Básica tanto no Brasil quanto internacionalmente. Os resultados mostram que há práticas realizadas em ambiente de programação em atividades extracurriculares, especialmente no contraturno e em período de férias, indicando que há ainda poucas práticas interligando o currículo com o App Inventor. Os resultados positivos têm relação com as estratégias metodológicas escolhidas para a aplicação das práticas, cujos melhores resultados estão relacionados aos interesses, aos problemas do cotidiano dos alunos e a partir da formação de professores das instituições. Por outro lado, as dificuldades apontadas envolvem limitações dos alunos em conhecimentos básicos e pouco tempo para o desenvolvimento das atividades.

Palavras-chave: Prática Pedagógica, Desenvolvimento de Aplicativo, Programação, Educação Básica.

\begin{abstract}
The teaching of computing in Basic Education has been presented as an alternative for the development of skills of the 21 st century. It is typically taught through programming languages such as App Inventor, a tool that enables the development of mobile applications for smartphones. However, we still do not have an understanding of the impacts that pedagogical practices have generated in student learning with this specific focus in the programming environment. The article presents the result of a Systematic Literature Mapping in which we sought the analysis of the impacts of pedagogical practices of application development with App Inventor in learning curricular contents of Basic Education both in Brazil and internationally. The results show that there are practices carried out in the programming environment in extra-curricular activities, especially in the extra-curricular shift and vacations period, indicating that there are still few practices linking the curriculum with App Inventor. The positive results are related to the methodological strategies chosen for the application of the practices, whose best results are related to the interests, to the daily problems of the students and from the training of teachers of the institutions. On the other hand, the mentioned difficulties involve limitations of the students in basic knowledge and little time for the development of the activities.
\end{abstract}

Keywords: Pedagogical Practice, Application Development, Programming, K-12. 


\section{Introdução}

O ensino de computação na Educação Básica vem sendo discutido mundialmente, e há um esforço para que seja consolidado nesta fase escolar (Hubwieser, 2012; Grover and Pea, 2013). Algumas iniciativas neste sentido já existem, como o Code.org, CodeClub, Computação na Escola e Meninas Digitais, entre outras, que visam desenvolver o Pensamento Computacional (Wing, 2006), uma habilidade importante do século XXI. Para promovê-lo, uma das formas encontradas por países europeus e alguns estados dos Estados Unidos (EUA) é o ensino da computação na Educação Básica por meio do uso de ambientes de programação visuais baseados em blocos, como o Scratch, o BYOB/Snap! e o App Inventor (Lye e Koh, 2014). A partir da analogia das peças de um quebra-cabeça, os blocos destes ambientes de programação só permitem o encaixe de uma peça a partir da correspondência de um código com a sintaxe correta o que, segundo aponta Valente (2016), facilita "o processo de descrição das instruções para a máquina, uma vez que a sintaxe das instruções é definida pelo encaixe dos blocos, contribuindo para minimizar esse tipo de erro, que é muito comum em linguagem de programação baseada na codificação de comandos" (Valente, 2016, p.874). Dentre os ambientes baseados em blocos, um dos mais utilizados é o App Inventor, que permite desenvolver aplicativos totalmente funcionais para smartphones e tablets. Com mais de 400.000 usuários ativos atualmente, presentes em 195 países, já foram criados quase 22 milhões de aplicativos por meio desta ferramenta (App Inventor, 2018). O conceito de aprendizagem móvel (mobile learning) se aproxima da perspectiva de desenvolvimento de aplicativos por estudantes na Educação Básica, apresentando um novo cenário para trabalhar a aprendizagem na ubiquidade, que é definido por Santaella (2013) como as formas de aprendizagem mediadas pelos dispositivos móveis.

Fantin (2015) defende que o significado da aprendizagem móvel se modificou a partir da cultura digital e os dispositivos junto à conectividade sem fio, que possibilitam mudanças didáticas na aprendizagem, ampliando assim as perspectivas de iniciativas com práticas pedagógicas com as mídias, no caso específico, no ambiente de programação.

De acordo com uma pesquisa realizada no Brasil em 2017 pelo Centro Regional de Estudos para o Desenvolvimento da Sociedade da Informação (CETIC), 93\% das crianças e adolescentes ( 9 a 17 anos) usam o telefone celular para acessar a internet e, segundo a mesma pesquisa, $84 \%$ acessam a internet nos telefones celulares, por meio de wifi. Tais resultados indicam que práticas pedagógicas com o uso de smartphones com crianças e adolescentes no Brasil são viáveis, por meio do acesso à internet nas escolas e dentro da perspectiva do BYOD (bring your own device), em que os smartphones dos alunos podem ser trazidos para a escola e utilizados pedagogicamente nas aulas. Com a propagação do uso de celulares e a partir das multifuncionalidades destes aparelhos, práticas pedagógicas com dispositivos móveis se tornam uma alternativa concreta para a sala de aula. Já existem algumas unidades instrucionais, como oficinas e cursos voltados ao ensino de computação usando App Inventor, além de tutoriais online, etc., o que nos mostra que ensinar computação por meio de desenvolvimento de apps é uma alternativa para práticas pedagógicas em ambientes de programação nas Instituições de ensino. A partir desta perspectiva, se propõe a conhecer, a partir de um mapeamento sistemático de literatura, quais os impactos do uso deste ambiente de programação sobre a aprendizagem na Educação Básica.

Deste modo, a revisão de literatura aqui apresentada teve como principal objetivo servir de caminho para responder à questão: Quais análises apresentam os impactos de práticas pedagógicas de desenvolvimento de aplicativos usando o ambiente de programação visual App Inventor, na aprendizagem de conteúdos curriculares da Educação Básica? 


\section{Metodologia}

A partir do objetivo proposto foi realizado um Mapeamento Sistemático da Literatura, que possibilita compreender o campo em que se insere a questão de pesquisa. Seguimos o processo proposto por Petersen et al. (2008), que afirma que "um mapeamento sistemático é um método definido de construir um esquema de classificação e estrutura em um campo de interesse", ou ainda, segundo Kitchenham (2007), uma "revisão ampla de estudos primários numa área específica que busca identificar que evidências estão disponíveis nessa área".

Os passos para o mapeamento foram definidos a partir da questão de pesquisa, assim como os critérios de inclusão e exclusão, fonte de dados, definição da string e depois a execução de busca, extração e análise dos dados, conforme detalhado a seguir:

2.1 Questão de pesquisa. Quais análises existem sobre o impacto na aprendizagem de conteúdos curriculares de forma interdisciplinar usando práticas pedagógicas envolvendo o desenvolvimento de aplicativos na Educação Básica?

A partir dessa questão de pesquisa foram refinadas as seguintes perguntas de análise:

PA1. Quais análises sobre o impacto do desenvolvimento de apps na Educação Básica foram publicadas?

PA2. Quais conteúdos curriculares e competências são avaliados nas análises?

PA3. Como as análises são feitas em relação ao tipo de pesquisa, coleta de dados, análise de dados, etc.?

PA4. Em quais contextos as análises foram realizadas?

PA5. Quais os principais resultados obtidos dessas análises?

2.2 Critérios de inclusão/exclusão. Para esta revisão, foram considerados artigos em língua portuguesa e inglesa com foco em práticas pedagógicas no contexto do ensino de computação na Educação Básica por meio da programação de apps usando o ambiente de programação App Inventor, buscando identificar os conteúdos curriculares nas produções. Dentre eles, foram excluídos artigos que focam em práticas no ensino superior, formação de professores e/ou outras linguagens de programação.

2.3 Fonte de dados. A busca foi realizada na base de dados Education Resources Information Center (ERIC), que possui referenciais com resumos e textos completos; na base Scopus, banco de dados de resumos e citações da literatura revisada por pares: periódicos científicos, livros e anais de congressos; no Google acadêmico, ferramenta de busca de literatura acadêmica que indexa um grande conjunto de dados em várias fontes diferentes (Haddaway et al., 2015) e ainda foram levados em consideração os artigos acessíveis pelo Portal de periódicos Capes, que oferece acesso aos textos completos de artigos.

2.4 Definição da string de busca. A string de busca tem como objetivo conectar conceitos que possam aproximar a área da educação e da computação, por conta da questão de pesquisa que envolve a prática pedagógica em um ambiente de programação. Minimizando o risco de omissão de artigos relevantes, incluímos também sinônimos aos principais conceitos relacionados com o foco da nossa pesquisa, conforme indicado na Tabela 1.

Tabela 1 - Strings de busca

\begin{tabular}{|l|l|}
\hline Fonte de Dados & String de busca \\
\hline Português & $\begin{array}{l}\text { ("educação básica" OR escola) AND ("dispositivo móvel” OR “aprendizagem móvel" OR "App Inventor") } \\
\text { AND ("pensamento computacional" OR "ciência da computação" OR programação) }\end{array}$ \\
\hline
\end{tabular}


Inglês

("K-12" OR school) AND ("mobile device" OR "mobile learning” OR "App Inventor") AND ("computational thinking" OR "computer science" OR programming)

Fonte: Elaborada pelas autoras (2018).

2.5 Execução de busca. As buscas foram realizadas no período de junho a agosto de 2018 pela primeira autora, sendo os resultados revisados pelas demais coautoras. Primeiramente foram identificados resultados potencialmente relevantes a partir da leitura do título e resumo e, em seguida, foram identificados os artigos relevantes com base na leitura detalhada de acordo com os critérios de inclusão/exclusão. Nas bases com muitos resultados, foram analisados somente os resultados mais relevantes, de acordo com o resultado da busca.

Dos resultados encontrados, muitos contemplam outras linguagens de programação, principalmente Scratch e, por isso, não são considerados relevantes para esta pesquisa. Encontramos também vários artigos abordando a formação de professores e práticas no Ensino Superior com App Inventor que, foram excluídos, por conta dos critérios de inclusão e exclusão.

\section{Resultados e Discussão}

Nessa seção serão analisadas e discutidas as informações extraídas dos artigos encontrados para cada uma das perguntas de análise.

\subsection{Quais análises sobre o impacto do desenvolvimento de apps na Educação Básica foram publicadas?}

Como resultado da pesquisa, foram identificados dez trabalhos como relevantes conforme apresentados na Tabela 2.

Tabela 2 - Informações sobre os artigos selecionados

\begin{tabular}{|c|c|c|}
\hline Citação & Referência bibliográfica & Descrição geral \\
\hline (Kim e Lee, 2017) & $\begin{array}{l}\text { KIM, S-W. LEE, Y. A study of educational method } \\
\text { using App Inventor for elementary computing } \\
\text { education. Journal of Theoretical \& Applied } \\
\text { Information Technology, } 95,4376-4384.2017 .\end{array}$ & $\begin{array}{l}\text { Atividade desenvolvida em uma escola na Coréia } \\
\text { do Sul no período de férias utilizando o ambiente de } \\
\text { programação App Inventor. }\end{array}$ \\
\hline $\begin{array}{l}\text { Chatzinik e } \\
\text { Papadaki, 2014) }\end{array}$ & $\begin{array}{l}\text { CHATZINIKOLAKIS, G. PAPADAKIS, S. } \\
\text { Motivating K-12 students learning fundamental } \\
\text { Computer Science concepts with App Inventor. } \\
\text { Anais da Int. Conference on Interactive Mobile } \\
\text { Communication Technologies and Learning, } \\
\text { Thessaloniki, Grécia, 2014. }\end{array}$ & $\begin{array}{l}\text { Apresentação aos alunos de conceitos } \\
\text { fundamentais de computação e programação, por } \\
\text { meio da utilização de todas as funções do App } \\
\text { Inventor. } \\
\text { O material desenvolvido pela equipe, também } \\
\text { contempla conteúdos de outras áreas de } \\
\text { conhecimento. }\end{array}$ \\
\hline $\begin{array}{l}\text { (A-Ghamdi et al., } \\
\text { 2016) }\end{array}$ & $\begin{array}{l}\text { A-GHAMDI, S. AL-RAJHI, A. N. A., N. AL-ONAIZY, } \\
\text { M., AL-KHALIFA H. S. Using App Inventor } 2 \text { in A } \\
\text { Summer Programming Workshop: Improvements } \\
\text { Over Previous Years. IEEE Global Engineering } \\
\text { Education Conference. Abu Dhabi, Arábia Saudita. } \\
2016\end{array}$ & $\begin{array}{l}\text { Aplicação de oficinas com meninas do Ensino } \\
\text { Médio ensinando programação no App Inventor. }\end{array}$ \\
\hline (Ni et al., 2016) & $\begin{array}{l}\text { NI, L. SCHILDER, D.SHERMAN, M. MARTIN, F. } \\
\text { Computing with a community focus: outcomes from } \\
\text { an app inventor summer camp for middle school } \\
\text { students. Anais do } 47^{\circ} \text { Simpósio Técnico da ACM } \\
\text { sobre Educação em Ciência da Computação. Memphis, } \\
\text { Tennessee, EUA, } 2016 .\end{array}$ & $\begin{array}{l}\text { Projeto e a avaliação ocorrido em uma semana } \\
\text { num acampamento de verão, com foco explícito na } \\
\text { criação de aplicativos a partir de necessidades da } \\
\text { comunidade local. }\end{array}$ \\
\hline (Vakil, 2014) & $\begin{array}{l}\text { VAKIL, S. A Critical Pedagogy Approach for } \\
\text { Engaging Urban Youth in Mobile App Development } \\
\text { in an After-School Program. Equity \& Excellence in } \\
\text { Education, 47(), 31-45, 2014. }\end{array}$ & $\begin{array}{l}\text { A partir da pedagogia crítica foram desenvolvidas } \\
\text { atividades em um projeto em que jovens } \\
\text { desenvolvem aplicativos voltados para questões } \\
\text { sociais. }\end{array}$ \\
\hline
\end{tabular}




\begin{tabular}{|c|c|c|}
\hline $\begin{array}{l}\text { (Mattos et al., } \\
2017)\end{array}$ & $\begin{array}{l}\text { MATTOS, M. S. XAVIER, F. C. PINTO, S. C. C. da S. } \\
\text { Uma análise sobre o uso de programação de jogos } \\
\text { para dispositivos móveis como recurso para o ensino } \\
\text { de matemática. Anais do Workshop do VI Congresso } \\
\text { Brasileiro de Informática na Educação. Recife, Brasil, } \\
2017 .\end{array}$ & $\begin{array}{l}\text { Oficinas e minicursos planejados numa escola } \\
\text { técnica realizando uma atividade interdisciplinar } \\
\text { com matemática e informática, visando apoiar o } \\
\text { aprendizado de matemática e desenvolvimento do } \\
\text { Pensamento Computacional. }\end{array}$ \\
\hline $\begin{array}{l}\text { (Sedano et al., } \\
2017 \text { ) }\end{array}$ & $\begin{array}{l}\text { SEDANO, P. R. NOGUEIRA, I. V. FRAGA. S. V. B. } \\
\text { BORGES, M. Aplicação do MIT App Inventor como } \\
\text { ferramenta de apoio à aprendizagem. Anais do IX } \\
\text { Congreso Iberoamericano de Educación Científica, } \\
\text { Mendoza, Argentina, } 2017 .\end{array}$ & $\begin{array}{l}\text { Atividades desenvolvidas numa ONG abrangendo } \\
\text { conceitos iniciais de programação como: variáveis, } \\
\text { estruturas de controle, estruturas de decisão e listas. } \\
\text { Os aplicativos escolhidos tinham relação com o } \\
\text { interesse dos alunos e as suas experiências atuais. }\end{array}$ \\
\hline $\begin{array}{l}\text { (Bauer et al., } \\
2017 \text { ) }\end{array}$ & $\begin{array}{l}\text { BAUER, R. D., FLORES, G. L. M., CRESTANI, A. V., } \\
\text { MOMBACH, J..Projeto codIFic@ @: Oficinas de } \\
\text { Programação em Dispositivos Móveis no Ensino } \\
\text { Fundamental. Anais de VI Congresso Brasileiro de } \\
\text { Informática na Educação. Recife, Brasil, } 2017 .\end{array}$ & $\begin{array}{l}\text { Reporta a experiência em um projeto de extensão } \\
\text { ao ensino de programação para adolescentes tendo } \\
\text { como metodologia o desenvolvimento do } \\
\text { Pensamento Computacional, Práticas Colaborativas } \\
\text { e Aprendizagem Baseada em Problemas. }\end{array}$ \\
\hline $\begin{array}{l}\text { (Gomes e } \\
\text { Melo, 2013) }\end{array}$ & $\begin{array}{l}\text { GOMES, T.C.S., De MELO, J.C.B. (2013) O } \\
\text { pensamento computacional no ensino médio: uma } \\
\text { abordagem Blended-Learning. Anais de XXI } \\
\text { Workshop sobre Educação em Computação. Maceió, } \\
\text { Brasil, 2013. }\end{array}$ & $\begin{array}{l}\text { Apresenta uma proposta metodológica baseada nas } \\
\text { premissas de aprendizagem significativa, a qual } \\
\text { delineia uma estratégia de ensino do Pensamento } \\
\text { Computacional de modo contextualizado, } \\
\text { incorporando técnicas blended learning para } \\
\text { promover um cenário mais flexível, bem como a } \\
\text { apresentação dos conteúdos de uma maneira mais } \\
\text { atraente e lúdica. }\end{array}$ \\
\hline $\begin{array}{l}\text { (Silva et al., } \\
2018)\end{array}$ & $\begin{array}{l}\text { SILVA, M. M. MIORELLI, S. T. KOLOGESKI, A. L. } \\
\text { Estimulando o pensamento computacional com o } \\
\text { projeto logicando. Revista: Observatório, 4(3), } 2018 .\end{array}$ & $\begin{array}{l}\text { O projeto tem como finalidade oferecer oficinas } \\
\text { lúdicas para os alunos de } 8^{\circ} \text { e } 9^{\circ} \text { anos do Ensino } \\
\text { Fundamental, a fim de trabalhar e desenvolver o } \\
\text { Pensamento Computacional, por meio do uso de } \\
\text { múltiplas ferramentas da Tecnologia da Informação } \\
\text { e Comunicação (TIC). }\end{array}$ \\
\hline
\end{tabular}

Fonte: Elaborada pelas autoras (2018).

As publicações a respeito de práticas pedagógicas envolvendo o desenvolvimento de aplicativos são recentes. Dos artigos relevantes encontrados, o mais antigo é de 2013, três anos após o lançamento do App Inventor, que aconteceu em 2010. Dentro dos critérios pré-estabelecidos, cinco publicações são nacionais e cinco são internacionais, e, em sua grande maioria, foram publicados por pesquisadores da área da Ciência da Computação. Tal dado mostra que, numa perspectiva interdisciplinar, o diálogo entre diferentes áreas de conhecimento pode contribuir com pesquisas sobre aprendizagem em ambiente de programação envolvendo o desenvolvimento de aplicativos na Educação Básica.

As propostas apresentadas nestes artigos são desenvolvidas em oficinas, minicursos e atividades em instituições. As práticas são organizadas de forma interdisciplinar (Mattos et al., 2017) e outras implicitamente envolvem conteúdos curriculares, a partir dos aplicativos produzidos em que é possível identificar áreas de conhecimento específicas (Chatzinik e Papadaki, 2014) e (Bauer et al., 2017).

O desenvolvimento do Pensamento Computacional é citado nos cinco artigos em língua portuguesa, com destaque para Sedano et al. (2017), que pontuam o ambiente de programação App Inventor auxiliando na aprendizagem por meio do desenvolvimento do Pensamento Computacional, numa perspectiva de contribuição em diferentes disciplinas da educação formal. Considerando que "o Pensamento Computacional pode trazer ao processo de ensino-aprendizagem a possibilidade de reorganizar as matrizes curriculares dos programas escolares de forma que estes apoiem o desenvolvimento das habilidades cognitivas, sociais e profissionais desejadas" (Sedano et al., 2017).

\subsection{Quais conteúdos curriculares/competências são avaliados nas análises?}

Quanto ao currículo escolar brasileiro, o mapeamento mostra que a disciplina de matemática se destaca nas práticas interdisciplinares com o uso do App Inventor, 
mesmo que alguns artigos não a especifiquem diretamente. Na pesquisa de Chatzinik e Papadaki (2014), por exemplo, o desenvolvimento de aplicativos é apresentado como motivador à aprendizagem de conceitos de programação. O material publicado das atividades desenvolvidas, e que está disponibilizado online ${ }^{1}$, mostra que os aplicativos desenvolvidos têm conteúdos de matemática e geografia.

Em outro artigo, Vakil (2014) traz a perspectiva da pedagogia crítica em práticas de desenvolvimento de aplicativos, numa atividade dialógica, indicando Freire (2000) como referência na ação educativa. Em seu estudo o autor apresenta a ideia da fluência computacional como um direito civil ${ }^{2}$, indicando a teoria de três dimensões de engajamento: comportamental, emocional e cognitivo. Nesta perspectiva, as atividades ocorrem a partir de diálogos e registros, abordando a justiça social com uma pesquisa etnográfica em um trabalho de campo com abordagem interpretativa.

No que diz respeito ao conhecimento das mídias, o ensino de computação é apresentado no estudo de Vakil (2014) como algo que contribui para o desenvolvimento de habilidades, que superam as necessidades de inserção no mercado de trabalho, aproximando os estudantes de conhecimentos que são importantes para inclusão na sociedade.

Em outro artigo, Silva et al. (2018) indicam uma proposta para o desenvolvimento de habilidades e competências para resolução de problemas do cotidiano e Chatzinik e Papadaki (2014) abordam o desenvolvimento de habilidades de pensamento crítico, a partir das atividades. Porém, observa-se uma falta de avaliações mais específicas, por exemplo, avaliando o impacto nas habilidades do século XXI. Mesmo citando a relevância desse aspecto, não há evidência da realização desta avaliação.

Os conteúdos curriculares nem sempre são o principal para aprendizagem dos alunos na era digital, porém, a integração dessas disciplinas escolares com outras áreas de conhecimento pode ampliar as competências e as habilidades dos alunos, por meio de estratégias metodológicas conectadas com as Tecnologias Digitais da Informação e Comunicação (TDIC), contribuindo com novas possibilidades de aprendizagem.

\subsection{Como as análises são feitas em relação ao tipo de pesquisa, coleta de dados, análise de dados, etc.?}

A maioria dos artigos não apresenta de maneira detalhada a metodologia adotada em cada uma das pesquisas. Inferindo essas informações, observa-se que elas variam em termos de rigor, duração etc., já que existem registros de pesquisas desenvolvidas desde o período de uma semana até o de um ano. Além disso, as pesquisas também variam quanto à sua natureza, sendo encontrados três estudos de casos, um estudo etnográfico, dois relatos de experiência e um projeto; em três casos, tal identificação não foi possível. No entanto, em comum percebe-se que em quase todas elas foram aplicados, na coleta de dados, prés e pós-testes, com exceção de Vakil (2014).

Tabela 3 - Características das análises

\begin{tabular}{|l|l|l|l|}
\hline Citação & Research design & Duração do estudo & Método(s) de coleta de dados \\
\hline Kim e Lee, 2017) & Estudo de caso & NI & $\begin{array}{l}\text { Pré e pós-teste } \\
\text { Teste de psicologia (escala likert) usado para } \\
\text { pesquisa de opinião. }\end{array}$ \\
\hline $\begin{array}{l}\text { Chatzinik e } \\
\text { Papadaki, 2014) }\end{array}$ & Estudo de caso & 4 semanas & $\begin{array}{l}\text { Questionários pré e pós-teste preenchidos pelos } \\
\text { alunos participantes e entrevistas } \\
\text { semiestruturadas com os professores }\end{array}$ \\
\hline
\end{tabular}

\footnotetext{
1 http: //www.sepchiou.gr/ai

2 Direitos e obrigações do indivíduo na sociedade.
} 


\begin{tabular}{|c|c|c|c|}
\hline & & & participantes. \\
\hline $\begin{array}{l}\text { (A-Ghamdi et al., } \\
\text { 2016) }\end{array}$ & $\begin{array}{l}\text { Relato de experiência } \\
\text { (ad-hoc) }\end{array}$ & 6 dias & Pré e pós-teste \\
\hline (Ni et al., 2016) & NI & $\begin{array}{l}1 \text { semana, } 6 \text { horas por dia num } \\
\text { total de } 30 \text { horas }\end{array}$ & Pré e pós-pesquisa e entrevista semiestruturada. \\
\hline (Vakil, 2014) & $\begin{array}{l}\text { Abordagem interpretativa } \\
\text { e etnográfica }\end{array}$ & 1 semestre & Entrevista aos alunos. \\
\hline (Mattos et al., 2017) & Estudo de caso & $\begin{array}{l}\text { Fase 1: Preparação e realização } \\
\text { das oficinas e minicurso } \\
\text {-Oficina: } 2 \text { meses } \\
\text {-Minicurso: } 5 \text { horas } \\
\text { Fase 2: } \\
3 \text { meses }\end{array}$ & $\begin{array}{l}\text { Aplicação de questionários com os alunos em } \\
\text { todas as etapas do projeto. }\end{array}$ \\
\hline $\begin{array}{l}\text { (Sedano et al., } \\
2017)\end{array}$ & NI & 5 encontros & $\begin{array}{l}\text { Os alunos responderam a } 2 \text { questionários on- } \\
\text { line para avaliar os conteúdos aprendidos nas } \\
\text { atividades. }\end{array}$ \\
\hline $\begin{array}{l}\text { (Bauer et al., } \\
\text { 2017) }\end{array}$ & $\begin{array}{l}\text { Relato de experiência } \\
\text { descritivo }\end{array}$ & 3 meses & $\begin{array}{l}\text { Dois diferentes questionários sendo um } \\
\text { aplicado aos alunos participantes do projeto e } \\
\text { outro a equipe executora. }\end{array}$ \\
\hline $\begin{array}{l}\text { (Gomes e Melo, } \\
2013)\end{array}$ & $\mathrm{NI}$ & $\mathrm{NI}$ & Questionário avaliativo \\
\hline $\begin{array}{l}\text { (Silva et al., } \\
\text { 2018) }\end{array}$ & Projeto (ad-hoc) & 2 semestres & $\begin{array}{l}\text { Em cada oficina é aplicado um pré-teste aos } \\
\text { alunos, com a finalidade de verificar o } \\
\text { conhecimento prévio, e um pós- teste para } \\
\text { averiguar se a oficina atingiu a meta proposta, } \\
\text { avaliando o desempenho dos alunos envolvidos } \\
\text { e a prática pedagógica aplicada. }\end{array}$ \\
\hline
\end{tabular}

Fonte: Elaborada pelas autoras (2018).

\subsection{Em quais contextos as análises foram realizadas?}

Os artigos encontrados apresentam análises em diversos contextos, tanto em relação ao nível educacional quanto às instituições em que são aplicadas, podendo ser em uma ONG ou em uma colônia de férias (acampamento de verão). A grande maioria é aplicada no contraturno escolar em formato de oficinas e minicursos. Uma das práticas (Kim e Lee, 2017) é realizada nas férias dos alunos, com o objetivo de não "prejudicar" a educação formal e para evitar que os alunos se distraiam com outros assuntos. Quanto a pesquisas vinculadas aos conteúdos curriculares, Mattos et al. (2017) abordam aplicação numa prova de matemática, Silva et al. (2018) relatam professores passando por uma formação e em seguida aplicando atividades em oficinas, minicursos ou em aulas regulares, junto com uma equipe multidisciplinar.

Quanto ao gênero feminino e masculino, há dentre os artigos analisados um estudo específico, realizado só com meninas na Arábia Saudita (A-Ghamdi et al., 2016), tendo como objetivo de encorajar meninas a participarem de oficinas de desenvolvimento de aplicativos. Essa proposta vem ao encontro de algumas outras iniciativas como Technovation Challenge ${ }^{3}$ e Meninas Digitais ${ }^{4}$, que visam desmistificar a área da tecnologia como um espaço masculino. 
A figura apresenta a quantidade de alunos participantes das atividades analisadas.

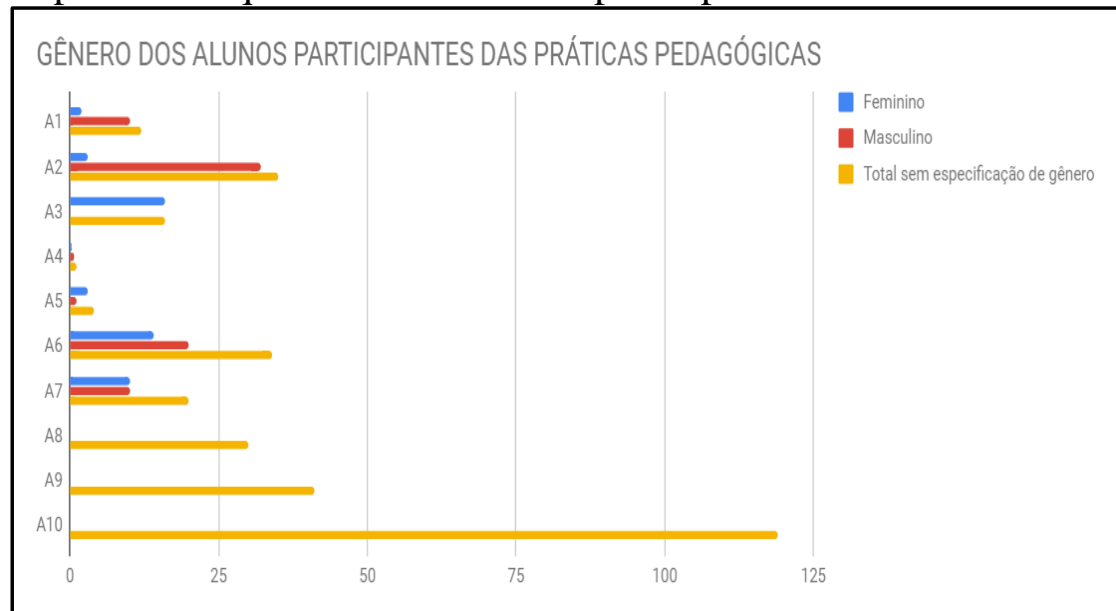

Figura 1 - Gênero dos alunos participantes das práticas pedagógicas.

Mesmo sem o registro de gênero das pesquisas de Bauer et al. (2017), Gomes e Melo (2013) e Silva et al. (2018) é possível identificar que a maioria dos participantes nos estudos, é de meninos, refletindo a percepção dessa área atualmente.

\subsection{Quais os principais resultados obtidos dessas análises?}

Os resultados apontam que alguns artigos apresentam propostas interdisciplinares, abordando temáticas curriculares com práticas pedagógicas no ambiente de programação App Inventor. Chatzinik e Papadaki (2014) produziram um material didático para as oficinas de desenvolvimento de aplicativos com exercícios envolvendo várias áreas de conhecimento. Eles afirmam que nas escolas gregas o uso dos dispositivos móveis para a aprendizagem ainda não foi examinado profundamente, mas reconhecem que, em pesquisas realizadas com o App Inventor houve melhora de conhecimentos quanto à programação.

Kim e Lee (2017) indicam que houve limitações no estudo em relação ao tempo de desenvolvimento de atividades, mas não é possível identificar no estudo qual foi o tempo reservado para aplicação das atividades com os alunos, para uma possível comparação com outros estudos.

Observa-se também que em três estudos o papel do professor é destacado nas práticas pedagógicas (Mattos et al., 2017) (Bauer et al., 2017) (Silva et al., 2018). Mattos et al. (2017) apresentam, no estudo, o professor envolvido na mediação e na avaliação das atividades realizadas esclarecendo dúvidas, propondo alternativas e projetos em parceria; no resultado dos questionários de Bauer et al. (2017) aparecem os professores conseguindo ensinar a programação, explicando o conteúdo de uma maneira engraçada e envolvente, e Silva et al. (2018) indicam professores presentes, participantes e auxiliando alunos, na perspectiva de futuros multiplicadores do projeto.

Ni et al. (2016), Chatzinik e Papadaki (2014) e Vakil (2014) indicam que trabalhos com temáticas de interesse dos alunos e resolução de problemas trazem resultados positivos. Ni et al. (2016) indicam que os alunos se tornam mais confiantes na criação de aplicativos abordando a resolução de problemas da comunidade. Chatzinik e Papadaki (2014) concluem que "o App Inventor e os dispositivos móveis, combinados com atividades focadas nos problemas do mundo real e nos interesses dos alunos, fornecem uma plataforma autêntica com grande potencial de aprendizado e devem ser seriamente considerados como um meio de atrair estudantes para o campo da computação" (Chatzinik e Papadaki, 2014, p.158). 
Vakil (2014) traz uma perspectiva da pedagogia crítica com uma alternativa para atividades significativas para os alunos, utilizando a programação e o design de um aplicativo móvel por meio de uma investigação sociopolítica de uma questão baseada na comunidade com a qual os alunos pudessem se relacionar por meio de suas experiências vividas. Essa perspectiva aproxima o conhecimento prévio e os interesses dos alunos, estimulando a construção de novos conhecimentos, que se encaixa com a Zona de Desenvolvimento Proximal, que "é a distância entre o nível de desenvolvimento real, que se costuma determinar através da solução independente de problemas, e o nível de desenvolvimento potencial, determinado através da solução de problemas sob a orientação de um adulto ou em colaboração com companheiros mais capazes" (Vygotsky, 1991, p.97).

$\mathrm{Ni}$ et al. (2016) apontam que o App Inventor pode ser usado para democratizar a computação, ampliando o uso da computação a todos os alunos, não apenas a uma minoria. Vakil (2014), quanto à pedagogia crítica, argumenta que "a fluência computacional, que engloba o conhecimento em mídia digital, engenharia, design thinking e Ciência da Computação, também é uma questão de direitos civis. Não só a fluência computacional é importante para obter acesso a oportunidades de educação e emprego, também é essencial para se tornar um cidadão ativo e informado, participante da sociedade" (Vakil, 2014, p.32). Este tipo de abordagem contribui, por ter elementos significativos no processo de letramento digital na formação do cidadão, no caso do aluno da Educação Básica, oportunizando a equidade social.

\section{Ameaças à validade}

O Mapeamento Sistemático de Literatura pode sofrer viés comum a partir dos resultados positivos, tendo maior probabilidade de serem publicados do que os negativos. No entanto, consideramos que os resultados dos artigos, sejam positivos ou negativos, têm apenas uma pequena influência sobre esse mapeamento sistemático. Outro risco é a omissão de estudos relevantes. A fim de reduzir esse risco, tivemos cuidado com a construção da string de busca, considerando não apenas os principais conceitos, mas também sinônimos. Ameaças quanto à seleção e à extração de dados foram aplacadas por meio de uma definição dos critérios de inclusão/exclusão. A extração de dados foi prejudicada em alguns casos, pelo fato que as informações relevantes nem sempre foram apresentadas explicitamente e/ou usando uma terminologia comumente aceita e, portanto, em alguns casos necessitando ser inferidas.

\section{Conclusão}

Os resultados mostraram que há práticas pedagógicas em ambiente de programação realizadas em sua maioria no contraturno escolar e em período de férias, indicando que há poucas práticas interligando conteúdos curriculares com App Inventor.

Os resultados positivos nas pesquisas têm relação com as estratégias metodológicas escolhidas para a aplicação das práticas. Dentre os melhores resultados estão práticas voltadas para os interesses, os problemas do cotidiano dos alunos e a partir da formação de professores das instituições para participação e atuação na pesquisa.

Há indícios de que o uso de dispositivos móveis não representou nenhum problema na concentração dos alunos, possivelmente por conta das estratégias metodológicas. As dificuldades apontadas envolvem limitações em conhecimentos básicos dos alunos e pouco tempo para as atividades.

Em alguns estudos os pesquisadores consideram que práticas com App Inventor podem trazer elementos importantes no ambiente escolar, por meio do currículo e do 
desenvolvimento de habilidades dos alunos. Porém, os estudos analisados não trazem dados substanciais para consolidar essa perspectiva.

Dessa forma faz-se necessário o desenvolvimento de pesquisas que possam trazer amostras de práticas pedagógicas em ambiente de programação App Inventor de forma interdisciplinar e conectada ao currículo, indicando que aprendizagens fazem parte deste contexto.

\section{Agradecimentos}

Este trabalho foi apoiado pelo $\mathrm{CNPq}$ (Conselho Nacional de Desenvolvimento

Científico e Tecnológico), uma entidade do governo brasileiro focada no desenvolvimento científico e tecnológico.

\section{Referências Bibliográficas}

App Inventor. 2018. Disponível em: <http://ai2.appinventor.mit.edu/> Acesso em: 13 de abril de 2018.

CETIC. TIC Kids Online. 2017. Disponível em: <https://cetic.br/pesquisa/educacao/indicadores>. Acesso em: 21 de setembro de 2018. FANTIN, Mônica. Novos Paradigmas da Didática e a Proposta Metodológica dos Episódios de Aprendizagem Situada, EAS. Educação \& Realidade. 40(2), Porto Alegre, 2015.

GROVER S., PEA R. Computational Thinking in K-12 a Review of the State of the Field. Educational Researcher, 42(1), 38-43, 2013.

HADDAWAY, N. R. et al. The role of Google Scholar in evidence reviews and its applicability to grey literature searching. PloS one, 10(9), 2015.

HUBWIESER, P. Computer Science Education in Secondary Schools -- The Introduction of a New Compulsory Subject. ACM Transactions on Computing Education, 12(4), 16.USA. 2012.

LYE, S. Y., KOH, J. H. L. Review on teaching and learning of computational thinking through programming: What is next for K-12?. Computers in Human Behavior, 41(C), 51-61. 2014.

PETERSEN, P., FELDT, R., MUJTABA, S., MATTSON, M. Systematic mapping studies on software engineering. In: Anais he 12th Int.Conference on Evaluation and Assessment in Software Engineering, Apúlia, Itália, 2008.

SANTAELLA, Lúcia. Comunicação Ubíqua: repercussões na cultura e na educação; São Paulo: Paulus, 2013.

VALENTE, J. A. Integração do Pensamento Computacional no Currículo da Educação Básica: Diferentes Estratégias Usadas e Questões de Formação de Professores e Avaliação do Aluno. Revista e-Curriculum, 14(3), 2016.

VYGOTSKY, L. S. A Formação Social da Mente. Editora: Martins Fontes. $4^{a}$ edição. 1991. São Paulo.

WING, J. M. Computational thinking. Communications of the ACM, 49(3), 33, 2006. 